\title{
CONDUCTA EN LA SITUACION TRANSVERSA DEL FETO
}

\author{
Doctor Alvaro Velaseo Ch.
}

La situación transversa del feto es una anomalía que frecuentemente se presenta en los Servicios Obstétricos y que se acompaña de una alta mortalidad fetal y morbilidad materna.

La discrepancia en las conductas ha producido una gran confusión. El mejor conocimiento de la contractilidad uterina, de las causas de anoxia fetal, de las complicaciones, ha disminuído el riesgo materno-fetal.

El presente estudio hace un análisis de las conductas seguidas en el Instituto Materno Infantil "Concepción Villaveces de Acosta” en Bogotá, se analizan sus resultados y se hacen sugerencias para futuras observaciones.

\section{MATERIAL}

Se analizan 185 casos de pacientes ingresadas al Instituto en los años de ı960 у г96ı. De estas pacientes, 37 $(20 \%)$ ingresan con feto muerto y han sido, en su mayoría, atendidas fuera del Instituto por personal incompetente. Las 148 restantes, ingresan con feto vivo, aunque en muchas ocasiones, con los mismos antecedentes.

La mayoría de las pacientes son multigrávidas y algunas pocas primi- grávidas; su frecuencia, detállase en el cuadro $\mathrm{N}^{o}$ I. Como se aprecia en él, las primigrávidas son escasas. Entre éstas se han encontrado tres pacientes con embarazo múltiple, un caso con fibroma previo y un caso de útero cordiforme. En las restantes posiblemente existía alguna anomalía congénita del útero, pero su presencia pasó inadvertida.

CUADRO No 1

FRECUENCIA DE LA SITUACION TRANSVERSA SEGUN LA GRAVIDEz

\begin{tabular}{ccr}
\hline Gravidez & No de casos & Porcentaje \\
\hline I & I3 & $7,02 \%$ \\
II & 36 & $29,45 \%$ \\
III & 30 & $16,21 \%$ \\
IV & 27 & $14,81 \%$ \\
V & I7 & $9,18 \%$ \\
VI & I7 & $9,18 \%$ \\
VII & I9 & $10,20 \%$ \\
VIII y más & 24 & $12,90 \%$ \\
\hline
\end{tabular}

Durante los mismos años de esta revisión se atendió un total de $25.08 \mathrm{I}$ partos. La frecuencia de la situación transversa del feto es pues del $0.7 \%$. Según H. Vermelin (I) la frecuencia es de $0,3 \%$. Según Nubiola (2) es de $0,5 \%$. 
Entre nuestros casos se hicieron il4 cesáreas entre las pacientes ingresadas con feto vivo y 2I entre las pacientes ingresadas con feto muerto. Total de cesáreas 135 .

Durante los mismos años se hicieron 1.276 cesáreas, de donde resulta que un $10,06 \%$ de este número corresponde a intervenciones practicadas en pacientes con situación transversa del feto.

\section{ANALISIS ETIOLOGICO}

Se analizó la etiología de nuestros casos, la cual se detalla en el cuadro No 2. Llama la atención la alta frecuencia de cesárea previa como causante de la situación transversa. Esta alta frecuencia nos impide considerarla como simple coincidencia y es necesario concluír que una se deriva de la otra. Es una etiología nueva, surgida por los progresos en los métodos quirúrgicos. Es de presumir que la falla de la acomodación fetal se debe, en estos casos, a retracciones fibrosas del segmento inferior del útero.
La placenta previa y la gemeralidad tienen también una alta frecuencia en nuestra serie y coincide más o menos con otras descripciones. La mayor frecuencia está en la multiparidad, lo cual es un hecho favorable para quienes asumen conductas profilácticas durante la gestación.

En el cuadro № 2 se clasifican como prematuros aquellos casos en los cuales no existía ninguna otra causa o estado patológico aparente.

\section{COMPLICACIONES DE LA SITUACION TRANSVERSA}

Debido a la falta de educación de nuestras pacientes y a la ignorancia del personal que habitualmente las atiende en sus domicilios, un gran porcentaje ingresa a un Hospital cuando han surgido grandes complicaciones que, de carácter irreductible, ensombrecen nuestras cifras de mortalidad fetal.

La frecuencia de estas complicaciones está detallada en el cuadro $N^{0} 3$.

CUADRO No 3

FRECUENCIA DE LAS CAUSAS RTIOLOGICAS

\begin{tabular}{|c|c|c|c|c|c|}
\hline Etiología & No I.M.I. & Porcent./I.M.I. & $\begin{array}{l}\text { H. Vermelin } \\
\text { Francia (1) }\end{array}$ & Field (3) & Posner (15) \\
\hline Multiparidad & 72 & $40 \%$ & $90 \%$ & - & $66 \%$ \\
\hline Cesárea previa & 28 & $15,1 \%$ & - & - & - \\
\hline Gemelos & 26 & $13, \mathrm{~g} \%$ & $15 \%$ & $17 \%$ & - \\
\hline Prematurez & $2 \mathrm{I}$ & $\mathrm{I} I, 3 \%$ & II $\%$ & $29 \%$ & $47,8 \%$ \\
\hline Placenta previa & I 8 & $9,7 \%$ & - & $10 \%$ & II, $7 \%$ \\
\hline Estrechez pélvica & 4 & $2,1 \%$ & - & - & $2,7 \%$ \\
\hline Utero cordiforme & 3 & $\mathrm{I}, 6 \%$ & - & - & - \\
\hline Feto gigante & $\mathrm{I}$ & $0,9 \%$ & - & - & $0,9 \%$ \\
\hline Tumor previo & 4 & $2, \mathrm{I} \%$ & - & - & $\mathrm{I}, 8 \%$ \\
\hline Hidramnios & 2 & $\mathrm{I}, \mathrm{O} \%$ & - & - & - \\
\hline
\end{tabular}


CUADRO No 3

COMPLICACIONES DE LA SITUACION TRANSVERSA

Hipertonía uterina

Inminencia de ruptura

Ruptura uterina

Sufrimiento fetal

Procidencia del cordón

Infección ovular

Abruptio placentae

Otras

En el cuadro se incluyen, bajo el títulos de "otras" complicaciones le- sario tener en cuenta cuando se va a determinar una conducta, que muchas de estas complicaciones o causas son entidad patológica predominante al determinar el tratamiento adecuado. Su análisis debe ser muy esmerado y prolijo y sobrepesada su gravedad.

\section{CONDUCTAS OBSTETRICAS}

Las conductas obstétricas seguidas en nuestro servicio, de acuerdo a la vitalidad del feto, son las siguientes:

\begin{tabular}{cc} 
a) Cesárea & I 44 casos $(77,02 \%)$ \\
b) Versión Interna y Ext Pod. & $34 \quad(28,98 \%)$ \\
\hline Total & I48
\end{tabular}

ves, tales como edema del cuello, agotamiento materno, etc. Siempre las consideramos como casos complicados, para efectos de conducta, pues tienen antecedentes de mala atención fuera del Instituto y frecuentemente han recibido altas dosis de ocitócicos.

\section{PATOLOGIA ASOCIADA}

Un alto número de pacientes tienen una o más entidades patológicas asociadas a la situación transversa del
En un caso se practicó Histerectomía por placenta acreta.

En ningún caso se practicó versión externa o mixta.

En los casos tratados con Versión Interna no se produjo ninguna ruptura uterina que obligara a tomar medidas quirúrgicas.

En los casos que ingresaron con feto muerto, la conducta asumida fue:

a) Cesárea

b) Versión Interna y Extracción

c) Embriotomía

Total

$\begin{array}{lll}2 \text { I } & \text { casos } & (56,75 \%) \\ \text { I3 } " & (35,13 \%) \\ 3 & & (8,10 \%) \\ 37 & \end{array}$

feto; en ocasiones es su causa, en otras es una complicación derivada de ella Los cuadros 2 y 3 nos dan una idea de estos estados patológicos Es nece-
En cinco casos de ruptura uterina se practicó Histerorrafia a excepción de un caso en el cual se hizo Histerectomía total. 
En un caso de ruptura uterina causada por versión interna se hizo Histerorrafía.

\section{ANALISIS DE LOS RESULTADOS}

I. Pacientes con feto muerto al ingreso

Número total de casos:

Paridad: Multíparas Primíparas

Iํ Tratadas con Cesárea

Número de casos: $2 \mathbf{I}$.

Se adopta esta conducta en los casos complicados y aquellos que presentan asociación a otros estados patológicos distócicos, que, priman en cuanto a la conducta a seguir.

Las condiciones anómalas sobreagregadas, que obligaron a tomar conducta, fueron múltiples y se detallan en el cuadro $\mathrm{N}^{0}{ }_{4}$.

CUADRO No 4

CONDICIONES ANOMALAs EN PACIENTES QUE INGRESAN CON FETO MUERTO Y A QUIENES SE PRACTICO CESAREA O LAPARATOMIA

Hipertonía uterina 2 casos

Inminencia de ruptura uterina 7 Ruptura uterina Infección ovular Placentae previa (a)

Cesárea anterior Cesárea anterior Estrechez pélvica Abruptio placentae $2^{\text {" }}$ gemelo "abandonado" Otras

(a) Esta de por sí no es indicación de Cesárea pero se presenta en asociación a otros estados patológicos.
Como se observa, las complicaciones mayores se refieren a alteraciones de la contractilidad uterina, por exceso, llegando a su grado máximo, inminencia de ruptura y ruptura. En estas pacientes está formalmente contraindicada cualquier conducta por vía baja.

Mortalidad materna: nula.

Morbilidad materna:

Ileo paralítico

I caso

Shock (placenta previa)

Infección de herida

I

I,

Desgarro de arteria Uterina I "

En los casos de ruptura se hizo laparatomía. En cuatro de ellos se hizo Histerorrafía y en uno Histerectomía.

29 Tratadas con versión interna y extracción podálica.

$\begin{array}{cr}\text { Total de casos: } & \text { I2 } \\ \text { Paridad: Multíparas } & \text { I2 } \\ \text { Primíparas } & \text { I }\end{array}$

Se practica esta maniobra en las pacientes con feto muerto, cuando se cumplen todos los requisitos y no existe ninguna contraindicación. Requiere un cuidadoso estudio de las condiciones maternas y de la proporcionabilidad feto-materna. Las indicaciones están limitadas a casos de anomalía pura de la acomodación fetal, sin patología asociada.

Mortalidad materna: nula.

Morbilidad: I caso de paro cardíaco por complicación anestésica. Se hizo toracotomía y masaje cardíaco. La paciente salió en buenas condiciones. 
I caso de ruptura uterina en una paciente con antecedentes de mala atención fuera del servicio. No se hizo Cesárea por dificultades hospitalarias temporales. Se hizo luego laparotomía e histerorrafía.

I caso de desgarro del cuello. Sin importancia.

A excepción del caso de ruptura uterina ocurrida en una intervención descomedida, la Versión Interna y Extracción Podálica en los casos de feto muerto ha probado ser aceptable siempre que se llenen todos los requisitos y se tengan en cuenta las contraindicaciones.

Se cumplen estas condiciones en muy limitado número de casos pues las pacientes ingresan cuando ha llegado el período en que cualquier intervención por vía vaginal constituye un riesgo gravísimo para la madre. En muchos casos, la muerte del feto indica una alteración grave de la dinámica uterina lo que de por sí es una contraindicación para las maniobras versionadoras.

En un caso se practicó una conducta absolutamente errónea: paciente con ruptura uterina a quien se hizo Versión interna. Posteriormente se hizo laparotomía e histerorrafía.

\section{$3^{0}$ Tratados con embriotomía}

En tres casos se practicó embriotomía, sin ninguna complicación ni accidente.

Se utilizó el ambriótomo de Turpin y se hizo sección raquídea. Esta intervención fue practicada por personal de amplia experiencia y los casos fueron esmeradamente escogidos. La baja incidencia coincide con la de los autores franceses $H$. Vermelin y M. Ribon (I), un $7 \%$. Entre nosotros esta baja proporción se debe a cierta aversión que a ella se tiene, pero en especial al poco entrenamiento del personal para ejecutar la maniobra. Como dijera Dexeus Font: La versión interna se ejecuta raramente y se teme la ejecución de una fetotomía por el forzoso desentreno (4).

\section{Pacientes que ingresan con} feto vivo

Número total de casos: $\quad$ I48

Paridad: Multíparas

Primíparas

\section{I ${ }^{\circ}$ Tratadas con versión interna $y$ extracción podálica}

Se asume esta conducta de acuerdo a las pocas indicaciones que le restan:

a) Situación transversa del segundo gemelo.

b) Situación transversa en gran multípara, feto pequeño.

No se adopta esta conducta en casos de distocia asociada ni casos complicados, exceptuando algunos casos especiales según criterio del obstetra tratante.

Las indicaciones que indujeron a tomar esta conducta están discriminadas en el cuadro No 5 . 
Segundo Gemelo en Sit Transv.

Situación Trasversa pura

Situación Transversa. Procidencia cordón

Placenta previa lateral

Abruptio Placentae

Indeterminada

$\begin{array}{lc}\text { I7 } & \text { casos } \\ \text { I2 } & , \\ \text { I } & , \\ 2 & , \\ \text { I } & , \\ \text { I } & ,\end{array}$

Mortalidad materna: nula.

A excepción de un caso de morta-

Morbilidad materna: I caso de infección puerperal.

\section{Mortalidad fetal en casos tratados con versión y extracción}

La mortalidad fetal se refiere a I I casos entre un total de 34 versiones. Los resultados globales de la versión se encuentran en la tabla número 6 . lidad fetal del cual no tenemos relación de peso, todos los casos de mortalidad están por debajo de 2.500 grs., correspondiendo a un porcentaje del $52,63 \%$.

Por encima de 2.500 grs., la mortalidad es nula, aunque la poca incidencia impide sacar conclusiones definitivas.

CUADRO No 6

RESULTADOS DE LA VERSION INTERNA Y EXTRACCION PODALICA EN LAS PACIENTES INGRESADAS CON FETO VIVO

\begin{tabular}{|c|c|c|c|c|c|c|}
\hline \multirow[b]{2}{*}{ Peso } & \multirow[b]{2}{*}{ Sanos } & \multirow[b]{2}{*}{ Morbilidad } & \multicolumn{2}{|c|}{ Mortalidad } & \multirow{2}{*}{$\begin{array}{l}\text { Sin re- } \\
\text { sultado }\end{array}$} & \multirow[b]{2}{*}{ Total } \\
\hline & & & No & $\%$ & & \\
\hline $5 \mathrm{OI}-\mathrm{I} .000$ & - & - & - & - & 一 & - \\
\hline I.OOI - I.500 & - & 一 & 2 & $100 \%$ & - & 2 \\
\hline $1.501-2.0()$ & I & I & 5 & $55 \%$ & 2 & 9 \\
\hline $2.00 \mathrm{I}-2.50 \mathrm{U}$ & 5 & - & 3 & $37 \%$ & - & 8 \\
\hline $2.501-3.000$ & 7 & I & - & - & - & 8 \\
\hline $3.00 \mathrm{I}-3.500$ & 5 & - & - & - & - & 5 \\
\hline $3.05 \mathrm{I}-4.000$ & - & 一 & - & 一 & - & - \\
\hline Indeterminado & I & - & - & - & - & 2 \\
\hline Totales & I9 & 2 & II & $32,35 \%$ & 2 & 34 \\
\hline
\end{tabular}

Como puede verse en el cuadro № 6, la mortalidad en la versión interna es de ir casos, correspondiendo a un $32,35 \%$. Cuatro de estos casos presentaban sufrimiento fetal intraparto.
La mortalidad es mayor cuanto menor el peso.

Mortalidad fetal según la indicación

Exactamente la mitad de los casos de versión fue efectuada para el se. 
gundo gemelo en Sit. Transv. La mortalidad en estos casos corresponde a un porcentaje ligeramente mayor. Véase la relación de mortalidad e indicación en el cuadro No 7 . Ocho pacientes ingresan con sufrimiento fetal intraparto. Cuatro de estos mueren después de nacidos.
En los fetos mayores no hay mortalidad.

Se anota, como anteriormente se hizo, que la mortalidad asciende a medida que disminuye el peso. Entre los dos casos de mortalidad comprendidos entre I.500 y 2.000 grs., fi-

CUADRO No 7

RELACION DE MORTALIDAD E INDICACION DE LA VERSION

\begin{tabular}{lcc}
\hline \multicolumn{1}{c}{ Indicación } & No de casos & Mortalidad \\
\hline Situación Transversa pura & I2 & I \\
Segundo gemelo & I7 & I \\
Situación Transversa procidencia Cordón & I & 6 \\
Placenta previa lateral & 2 & I \\
Abruptio Placentae . . . . . & I & I \\
Indeterminada & I & I \\
\hline
\end{tabular}

Segundo gemelo y mortalidad en la versión interna

Se hizo versión en 17 casos de segundo gemelo. Dos de estos carecen de la respectiva historia del niño por cual no sabemos el resultado final. No los contamos en la mortalidad. En el cuadro No 8 se resume la mortalidad respecto al peso.

La mortalidad es mayor que la mortalidad global, aumenta en los fetos que pesan de 1.500 a 2.500 grs. gura un caso que ingresó al servicio 38 horas des pués del primer parto.

En el grupo de 2.500 grs. figura uno que ingresó dos horas después del primer parto.

Los tres casos restantes de mortalidad, corresponden a pacientes que ingresaron al comienzo del parto y fueron atendidas en su totalidad en el Instituto.

La mortalidad de los segundos gemelos se debe muy posiblemente a

CUADRO No 8

MORTALIDAD DEL ?o GEMELO CON VERSION INTERNA

\begin{tabular}{ccccc}
\hline Peso & Sanos & Morbil & No & Mortalidad \\
\hline I.00I - I.500 & - & - & I & I00 $\%$ \\
I.50I - -2.000 & I & - & 2 & $66,66 \%$ \\
$2.00 I-2.500$ & 4 & - & 3 & $42,87 \%$ \\
$2.501-3.000$ & 4 & - & - & - \\
\hline
\end{tabular}


un proceso de anoxia que se presenta después de la expulsión del primer feto, debida a desprendimiento de placenta de mayor o menos cuantía, proceso que será tanto más nocivo cuanto más dure este intervalo y cuanto menor sea el tamaño del feto. Para reducir este período de anoxia es recomendable verificar la versión y extracción lo más rápidamente po-
$8,5 \%$, (mortalidad en un feto que pesó I.500 grs.).

El número de casos es muy pequeño para derivar conclusiones definitivas, pero se puede pensar que la mortalidad es mayor con la reducción de peso. Las versiones efectuadas en niños a término tuvieron buen resultado. (Cuadro $\mathrm{N}^{0} 9$ ).

CUADRO No 9

RELACION DE MORTALIDAD DE LOS CASOS DE SITUACION TRANSVERSA TRATADOS CON VERSION. (SITUACION TRANSVERSA PURA)

\begin{tabular}{cccc}
\hline Peso & Sanos & Morbil & Mortal \\
\hline I.00I - I.500 & - & I & - \\
I.50I - 2.000 & - & I & - \\
$2.001-2.500$ & I & - & - \\
$2.501-3.000$ & 2 & 2 & - \\
$3.001-4.000$ & 3 & - & - \\
\hline
\end{tabular}

sible después de la expulsión del primer feto. La maniobra versionadora parece no influír en estos casos, en la mortalidad pues todos conocemos la facilidad con que se efectúa y la falta de traumatismo que la acompaña. No creemos que la Cesárea reduzca este riesgo.

Es posible reducir la mortalidad haciendo un esmerado cuidado del recién nacido, en la mayoría de los casos, prematuro.

\section{Situación transversa pura y Versión interna}

En esta serie llama la atención que sólo existe un caso de mortalidad entre doce versiones efectuadas en estas condiciones. Corresponde a un
Los casos fueron muy bien estudiados y se valoró al máximo su riesgo, de allí la escasa mortalidad.

Esto demuestra que cuando existe situación transversa pura sin complicaciones ni patología asociada, la versión puede ejecutarse con poco riesgo, es necesario un buen conocimiento de la patología dinámica antes de precipitarse a hacer versiones, de otra manera el resultado puede ser desastroso.

En los casos restantes en los cuales se efectuó la maniobra, la mortalidad fue total, lo cual indica que los casos de patología asociada son de mal pronóstico si se tratan con versión. Pero es necesario tener en cuenta que en esos casos el pronóstico fe- 
tal ya era muy pobre y por esta razón se adoptó la conducta por vía vaginal.

Son casos en los cuales ni aún la cesárea puede impedir la muerte fetal. Son fetos con un sufrimiento intraparto muy marcado y pueden considerarse casi perdidos sea cual fuere la conducta que se tome. Muchas veces se prefiere la versión para dar un mejor futuro obstétrico a la paciente.

Solamente se reduce esta mortalidad, adoptando conductas más tempranamente, evitando la prolongación del período del sufrimiento fetal. En la mayoría de nuestros casos esto $\mathrm{\epsilon}$ rasi imposible puesto que las pacientes ingresan en períodos avanzados del parto.

\section{Morbilidad fetal en los casos de pacientes tratadas con versión}

3 casos de signos de anoxia intraparto.

I caso de parálisis facial.

I caso de parálisis braquial.

I caso de bronconeumonía.

I caso de fractura del maxilar.

2. Pacientes con feto vivo al ingreso, tratadas con Cesárea.

Esta conducta es la más frecuentemente adoptada entre nosotros por tener un amplio margen de seguridad en todos los casos complicados o aquellos a los cuales se asocia un estado patológico (en muchos casos este estado patológico asociado prima sobre la situación transversa para efectos de la conducta) y porque de acuerdo a las normas modernas de la
Obstetricia, la versión interna y extracción podálica va cediendo campo a. la Histerotomía como conducta atraumática.

Se practicó en el Instituto en II4 casos. En todas las oportunidades se hizo Cesárea Segmentaria Transperitoneal.

Las indicaciones por las cuales se efectuó la cesárea fueron variadas y están anotadas en el cuadro № ro. Algunas pacientes presentaron una o más indicaciones; cuarenta y tres pacientes ingresaron con sufrimiento fetal, entre éstas. nueve fetos mueren después de la intervención.

\section{Resultado de los casos tratados con cesárea}

Mortalidad materna: nula.

Morbilidad materna:

Ileo paralíticopost-operatorio I caso Infección de la herida

Fiebre puerperal

Endometritis

Peritonitis

$5 "$
I4,
$2 \quad$,
I $"$

Se practicó una histerectomía por placenta acreta.

Debe anotarse que de este grupo, nueve pacientes habían sido operadas de cesárea por situación transversa en años anteriores. Seis pacientes repitieron situación transversa en el siguiente embarazo.

Dos pacientes de infección endometrial post-operatoria hicieron ruptura uterina en el embarazo siguiente, una de ellas ruptura espontánea. 
INDICACIONES DE LAS CESAREAS EFECTUADAS

\begin{tabular}{lcc}
\hline \multicolumn{1}{c}{ Indicación } & No de casos & Mortalidad \\
\hline Situación transversa pura & 40 & 4 \\
Antecedente de Cesárea & 27 & 2 \\
Hipertonía uterina & 4 & I \\
Inminencia de ruptura uterina & 7 & 0 \\
Procidencia de cordón & I4 & 3 \\
Placenta previa (a) & I 4 & 4 \\
Infección amniótica & 4 & - \\
Estrechez pélvica & 3 & - \\
Polihidramnios & 2 & - \\
Tumor previo & 4 & - \\
Utero cordiforme & 3 & I \\
Embarazo gemelar & 4 & 2 \\
Segundo gemelo en Sit. Trans. (b) & 2 & - \\
Operación de Manchester prev. & I & - \\
Inercia uterina & I & - \\
Feto gigante & I & - \\
Abruptio placentae & I & - \\
Otras & 5 & \\
\hline
\end{tabular}

(a) Tiene además, en un caso, antecedente de cesárea.

(b) Tiene antecedente de cesárea.

Mortalidad fetal en casos tratados con cesárea

La mortalidad fetal fue de i7 casos, lo cual corresponde a un porcentaje de $14,91 \%$. Los resultados globales de la cesárea aparecen en el cuadro $\mathrm{N}^{0}$ i I. Nueve casos presentaban sufrimiento fetal antes de la operación.

Como se vé en el cuadro, la mortalidad se presenta especialmente en los casos por debajo de $2.50 \mathrm{I}$ gr. En estos grupos hay I4 muertes o sea un porcentaje de $36,84 \%$. (No se tiene en cuenta dos casos cuyos resul- tados se desconocen y un caso de peso desconocido).

Por encima de 2.500 grs. hay dos muertos que corresponde al 2,66\%.

La mortalidad global es del $14,91 \%$ ( I7 casos).

La mortalidad es menor cuanto mayor es el peso fetal.

En dos casos en los cuales se practicó cesárea para el segundo gemelo, por antecedente de cesárea, los fetos murieron.

Por la comparación de los resultados salta a la vista que la cesárea reduce notablemente la mortalidad. 
La mortalidad se reduce en un person (5), según ellos, la mortali$17,44 \%$.

La mortalidad por debajo de 2.500 gramos se reduce en un $15,79 \%$.

Por encima de 2.500 grs., la mortalidad en los casos tratados con versión ha sido nula, pero debido al número tan reducido de casos no es posible comparar con los casos o porcentaje de los casos tratados con cesárea. En estos el porcentaje es mínimo.

La reducción de la mortalidad ha sido ya reportada por Harris \& Epdad se redujo del $33,3 \%$ a un $2 \%$. Aunque entre nosotros no es tan notoria, es un hecho significativo que debe tomarse en cuenta para el futura.

Desgraciadamente no podemos comparar nuestras estadísticas, punto por punto, con aquellas de países más adelantados pues el material humano y hospitalario es absolutamente diferente.

Aun con la cesárea la mortalidad es muy alta en comparación a la mortalidad de otro tipo de presentación.

CUADRO No 11

RESULTADO DE LOS CASOS TRATADOS CON CESAREA

\begin{tabular}{|c|c|c|c|c|c|c|}
\hline \multirow[b]{2}{*}{ Peso } & \multirow[b]{2}{*}{ Sanos } & \multirow[b]{2}{*}{ Morbilidad } & \multicolumn{2}{|c|}{ Mortalidad } & \multirow{2}{*}{$\begin{array}{l}\text { Sin re- } \\
\text { sultado }\end{array}$} & \multirow[b]{2}{*}{ Total } \\
\hline & & & No & $\%$ & & \\
\hline $50 \mathrm{I}-\mathrm{I} .000$ & - & - & I & $100 \%$ & - & I \\
\hline I.0OI - I.500 & - & $-\quad \therefore$ & 4 & $100 \%$ & 一 & 4 \\
\hline $\mathrm{I} .50 \mathrm{I}-2.000$ & 2 & 3. & 4 & $36 \%$ & 2 & I I \\
\hline $2.000 \mathrm{I}-2.500$ & 9 & 8 & 5 & $22 \%$ & - & 22 \\
\hline $2.501-3.000$ & $2 I$ & 5 & I & $3 \%$ & 一 & 27 \\
\hline $3.0001-3.500$ & $2 I$ & 8 & - & - & I & 30 \\
\hline $3.501-4.000$ & IO & I & I & $8 \%$ & $\mathrm{v}$ & 13 \\
\hline Indeterminado & 3 & I & I & - & I & 6 \\
\hline Totales & 66 & 26 & $\mathrm{I} 7$ & $\mathrm{I} 4,9 \mathrm{I} \%$ & 5 & II 4 \\
\hline
\end{tabular}

MORTALIDAD FETAL COMPARADA ENTRE LOS CASOS TRATADOS CON CESAREA Y VERSION INTERNA

CUADRO No 12

MORTALIDAD COMPARADA

\begin{tabular}{|c|c|c|c|c|c|c|c|c|c|}
\hline & \multicolumn{3}{|c|}{ Global } & \multicolumn{3}{|c|}{ Menos de 2.500} & \multicolumn{3}{|c|}{ Más de 2.500} \\
\hline & Ne & Mort. & $\%$ & No & Mort. & $\%$ & No & Mort. & $\%$ \\
\hline Versión & 34 & I I & 32,35 & I9 & IO & 52,63 & I3 & - & 0 \\
\hline Cesárea & I I4 & I7 & $\mathrm{I} 4,9 \mathrm{I}$ & 38 & I4 & 36,84 & 75 & 2 & 2,66 \\
\hline
\end{tabular}


La única manera de reducirla es actuando precozmente, evitando la presencia de la anoxia y sufrimiento fetal.

\section{Mortalidad comparada con otros análisis}

Aunque los medios son diferentes y las condiciones hospitalarias variables, hago una pequeña comparación con algunas cifras encontradas en la bibliografía americana y europea.

a) Mortalidad global en la situación transversa.

Field \& Nelson (3)

H. Demelin (I)

Eastman (8)

Eastman (8) ruptura prematura membranas

Gaviria (9)

Posner (I5)

I. M. I. (Presente estudio) $12 \%$ $40 \%$ $10 \%$

b) Mortalidad en casos tratados con versión.

\section{Gaviria (9)}

H. Demelin (I)

Kieva ( Iо) (En prematuros) $50 \%$

Dexeus Font (II)

Muñoz Agüero (I4)

Posner (15) r.000 a 2.500 grs. $21 \%$

Posner (15) 2.500 y más grs. $26 \%$

I. M. I. (Presente estudio) $32 \%$

c) Mortalidad en casos tratados con cesárea.

Muñoz Agüero (14)

Harris \& Epperson (5)

$3,7 \%$

Posner (I5) r.000 a 2.500 grs. $40 \%$

Posner (I5) 2.500 grs. y más $7 \%$

I. M. I. (Presente estudio I I $9 \%$ \% d) Mortalidad general de la cesárea (Indicaciones varias)

Delgado (I2 I. M. I.

Gaviria (9)

Down (I3)

$5,3 \%$ $27,5 \%$

$6 \%$

Las diferencias de las estadísticas son muy grandes debido al sistema empleado en la depuración de los casos de mortalidad, diferentes entre cada autor.

El presente estudio solamente hace exclusión de los fetos que ingresan muertos al Servicio.

La falta completa de autopsias impide hacer mayor depuración.

\section{COMENTARIOS}

Es indudable que en el Instituto ha mejorado notablemente el pronóstico materno. Anteriormente la situación transversa abandonada constituía una de las entidades de más alto porcentaje de mortalidad materna; especialmente cuando el cuadro se asociaba con complicaciones graves como ruptura e infección. En la actualidad, las mejores condiciones hospitalarias, mayor número de centros prenatales, mejor conocimiento de la contractilidad uterina, han influído decididamente en el mejor pronóstico materno.

La cesárea en los casos de feto muerto, cuando la paciente presenta contracción alterada por exceso, ha sido una de las mejores contribuciones para anular la mortalidad y las rupturas uterinas. La infección es mejor combatida con el espectro antibiótico. 
La mortalidad fetal es todavía muy alta, se debe esto especialmente a que las pacientes ingresan muy tardíamente al Instituto cuando las condiciones fetales son ya muy precarias.

Es obvio que la cesárea sea la intervención más adecuada para este tipo de patología, salvo casos debidamente escogidos, ideales para la versión, que están casi limitados a aquellas pacientes multíparas con feto pequeño, maduro, sin patología materna asociada, ni complicaciones y que llenan absolutamente todos los requisitos de la ejecución de la versión interna.

El obstetra debe pensar siempre que el hecho de haber practicado unas pocas versiones con éxito no lo autoriza para practicarla repetidamente sin agotar siempre todos los requisitos.

Otra entidad susceptible de versión interna es el segundo gemelo, en situación transversa, efectuada tempranamente, no más allá de quince minutos después de la expulsión del primer feto. No existe razón para prolongar un período anóxico cuando se tiene la absoluta seguridad de que el feto no puede nacer espontáneamente, y si tarde o temprano vamos a efectuar una versión lo mejor, lógicamente, será practicarla inmediatamente después de la expulsión del primer feto.

La alta mortalidad fetal por debajo de 2.500 grs. se debe a la poca resistencia de estos fetos a la anoxia y a los traumatismos, a la susceptibilidad excesiva a los anestésicos, a los cambios de presión materna, etc. Las escasas reservas defensivas, inadaptabilidad al medio externo, una vez nacidos, favorecen su patología y son víctimas de cualquier agresión física, química o biológica, con mayor razón cuando ha sufrido previamente un traumatismo grave; por esto la versión interna, no es muy recomendable en los prematuros y serán ellos quienes reciban con mayor beneficio las ventajas de la cesárea.

El pediatra debe comunicar al obstetra los resultados finales de los casos y el obstetra a su vez informar las condiciones previas al nacimiento, sólo así se progresará en el manejo del recién nacido, bienestar de la sociedad que surge en las manos de estos profesionales.

\section{PROGRAMA PARA LA REDUCCCION DE LA MORTALIDAD}

Un programa para reducir la mortalidad, que debe comprender tanto normas generales como limitadas a la entidad obstétrica que nos preocupa, debe llenar los siguientes puntos:

Iํ. Educación de la mujer en los problemas higiénicos generales y particularmente obstétricos, educación que debe iniciarse en los colegios y escuelas. Con participación de campañas oficiales de los Ministerios de Salud y Educación en combinación con la Universidad, la cual podría, con su personal estudiantil, propagar los beneficios profilácticos que tanto necesita nuestro pueblo. 
$2^{\circ}$. Establecimiento de un número suficiente de centros prenatales y hospitales de maternidad, con personal idóneo, especializado, para limitar progresivamente la acción funesta de las "comadronas".

3․ Manejo de la situación transversa del feto.

a) En prenatal, practicar versión externa a las pacientes multíparas cuyo único estado patológico obstétrico sea la posición fetal. Nunca hacer en primíparas. Debe efectuarse alrededor de las 36 semanas de gestación.

b) Durante el trabajo de parto.

$\mathrm{Si}$ la paciente ingresa con feto muerto deben tomarse las siguientes medidas:

Cesárea: En caso de existir patología distócica asociada o complicaciones graves.

Versión interna: Podrá efectuarse en casos esmeradamente escogidos en los cuales no exista patología asociada ni complicaciones, deben reunir estrictamente todos los requisitos de la versión (Tonicidad uterina normal, membranas íntegras, dilatación total del cervix, feto de tamaño pequeño. No se hará en primíparas).

Embriotomía: Es la conducta adecuada en las pacientes que ingresan con feto muerto, y que no presentan ninguna patología asociada ni complicación grave alguna, debe ser siempre efectuada por personal de amplio entrenamiento. Es la conducta adecuada especialmente cuando se trata de primíparas y casos con infección ovular.

Si la paciente ingresa con feto vivo, la cesárea es la conducta de preferencia en todos los casos.

La versión interna, seguida o no de extracción podálica se practicará en el segundo gemelo y en algunos casos de multíparas a término en los cuales no se encuentra patología asociada ni complicaciones y que reúnen todos los requisitos para practicarla sin riesgos.

Excepcionalmente se adopta la conducta versionadora en lugar de cesárea cuando el feto está vivo, pero su vitalidad está tan comprometida que no se justifica la operación. Son casos en los cuales particularmente el pronóstico fetal está absolutamente perdido.

En los casos en que el feto sea menor de r.ooo grs. se hará versión en lugar de cesárea hasta tanto las condiciones hospitalarias sean suficientes para permitir una mayor supervivencia de estos niños. Es claro que esta conducta tendrá necesariamente que cambiar a medida que progresen nuestros cuidados al prematuro.

Los casos en los cuales existe ruptura uterina se tratarán con histerorrafía ó histerectomía de acuerdo al tipo, tiempo, sitio de la ruptura, según el estado de vitalidad de los tejidos y según la presencia o ausencia de infección grave (6-7).

La paridad de la paciente, número de hijos vivos, edad, estado general 
de la paciente, facilidades hospitalarias, son factores que influyen también en la adopción de la operación a efectuar.

Existen casos especiales, aislados que no pueden agruparse para efectos de la conducta y ésta deberá tomarse independientemente, según el tipo de patología. Tal es el caso de pacientes con grave infección ovular y ute- rina en las cuales se efectuará una Histerectomía global.

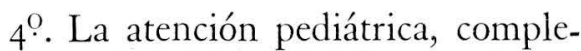
mento definitivo de las conductas anteriores debe iniciarse desde el momento del nacimiento y se continuará esmeradamente en la sala-cuna con especial preocupación sobre las medidas de atención en los casos de prematuros.

\section{BIBLIOGRAFIA}

1. VERMELIN H., RIBON M.: Presentation de l'epaule. Encyclopedie Medico Chirurgicale. París, 1960.

2. NUBIOLA, ZARATE: Tratado de Obstetricia. Ed. Labor. 1951.

3. FIElD \& NELSON. Errors of presentation. Am. J. Obst. \& Gynec. Vol. 78. No 3. 1959.

4) DEXEUS FONT: Influencia de la conducta obstétrica sobre la mortalidad perinatal. Progresos de Obstetricia y Ginecología. Vol. IV. Fasc. III. 1961. Abst.

5. HARRIS \& EPPERSON. An analysis of 131 cases of Transverse presentations. Am. J. Obst. \& Gynec. 59:1105. 1950.

6. MATTEWS: Uterine rupture. Year Book of Obstetrices \& Gynecology. 1961-1962. Abst.

7. VAlenzuela, MAtarriaga: Rupturas uterinas. Year Book of obstetrics \& Gynecology. 1961-1962. Abst.

8. eastman, Helman: Williams obstetrics. Apleton-Century Fox. 1961.

9. GAVIRIA B. COMITE DE LETALIDAD: Letalidad gerinatal. Revista Colombiana de Obstetricia y Ginecología. Vol. XI. No 3. 1960.

10. MAKEIEva O.: Profilaxis de las enfermedades de la madre y del recién nacido. Edición en lenguas extranjeras. Moscú. 1959.

11. DEXEUS FONT.: Tratado de Obstetricia. Salvat Ed. 1957.

12. DElgado E.: Mortalidad perinatal en cesárea. Revista Colombiana de obstetricia y Ginecología. Vol. XII. No 5.

13. DOWNS \& KURLECZ. Perinatal Mortality. Am. J. Obst. \& Gynec. Vol. 77. No 3.1959.

14. MUÑoz AGÜERO: Las situaciones transversas. Progresos de Obstetricia y Ginecología. Vol. IV. Fasc. 1961.

15. FOSNER et al.: The transverse lie. Am. J. Obst. \& Gynec.. Vol. 83. No 2.1962. 